\title{
Carbon monoxide concentration in donated blood: relation to cigarette smoking and other sources
}

\author{
Anna-Maja Åberg, Birgitta Nilsson Sojka, Ola Winsö, Pernilla Abrahamsson, Göran Johansson, and \\ Jan Erik Larsson
}

BACKGROUND: Carbon monoxide (CO) is normally present in the human body due to endogenous production of CO. CO can also be inhaled by exposure to external sources such as cigarette smoke, car exhaust, and fire. The purpose of this study was to investigate CO concentrations in blood from 410 blood donors at the blood center in Umeå, Sweden. To further evaluate the effects of cigarette smoking on CO concentrations, the elimination time for $\mathrm{CO}$ was examined in six volunteer smokers after a smoked cigarette.

STUDY DESIGN AND METHODS: Blood samples from whole blood donors were obtained during the blood center's routine operation. In connection with blood donations, demographic and behavioral data were collected from the donors. The CO concentration was determined using gas chromatography.

RESULTS: The majority of blood donors had approximately the same CO concentration (mean, $84.5 \mu \mathrm{mol} /$ L). In 6 percent of the samples, the concentrations were higher than $130 \mu \mathrm{mol}$ per L. The highest CO concentration was $561 \mu \mathrm{mol}$ per $\mathrm{L}$. The main source for these high $\mathrm{CO}$ concentrations appeared to be cigarette smoking. In the volunteer smokers, the elimination time after a smoked cigarette varied significantly, with elimination half-lives from 4.7 to 8.4 hours.

CONCLUSION: These results show that blood bank red blood cell bags may have CO concentrations above the physiologic level. The time interval between cigarette smoking and blood donation seems to be a particularly important factor for elevated $\mathrm{CO}$ concentrations.

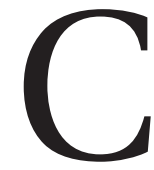
arbon monoxide (CO) is formed in the body during degradation of hemoglobin ( $\mathrm{Hb})$. Approximately $12 \mathrm{~mL}$ of $\mathrm{CO}$ is formed every day, bringing about a carboxyhemoglobin (COHb) per total $\mathrm{Hb}$ fraction of 1 to 2 percent. ${ }^{1}$ This level can be elevated as a result of contributions from external sources like cigarette smoke, car combustion, fire, or other sources of incomplete combustion. CO has higher affinity for $\mathrm{Hb}$ than oxygen. When bound to $\mathrm{Hb}$, it not only displaces oxygen, but also shifts the oxygen dissociation curve to the left making it harder for oxygen to be released in tissues. ${ }^{2}$ CO is also toxic in the mitochondria since it alters cytochrome $c$ oxidase in the respiratory chain. ${ }^{3}$ A case report in a 14-month-old child describes how cardiopulmonary bypass with a red blood cell (RBC)primed circuit, which was later found to contain 7.2 percent $\mathrm{COHb}$, increased the patient $\mathrm{COHb}$ to 3.7 percent when cardiopulmonary bypass was initiated. ${ }^{4}$ This potential risk of impaired oxygen delivery was successfully reversed by administration of 100 percent inspired oxygen, but the authors concluded that the incident could potentially have resulted in a dangerous situation for the child after termination of cardiopulmonary bypass if it went undetected and untreated.

ABBREVIATIONS: $\mathrm{COHb}=$ carboxyhemoglobin; $\mathrm{ROC}=$ receiver operating characteristic.

From Anesthesiology and Intensive Care Medicine and Transfusion Medicine, Umeå University Hospital, Umeå, Sweden.

Address reprint requests to: Anna-Maja Åberg, Department of Surgical and Perioperative Sciences, Division of Anesthesiology and Intensive Care, Umeå University Hospital, SE-901 87 Umeå, Sweden; e-mail: annamaja.aberg@anestesi.umu.se.

Financial support from the Medical Faculty, Umeå University, is acknowledged. The authors declare no conflict of interest.

Received for publication May 7, 2008; revision received August 18, 2008; and accepted August 19, 2008.

doi: 10.1111/j.1537-2995.2008.01951.x

TRANSFUSION 2009;49:347-353. 
Elevations in $\mathrm{COHb}$ levels in banked blood may be of clinical importance. Cigarette smoking has been found to be a major source of $\mathrm{CO}$ in banked blood. ${ }^{5}$ In one study, $\mathrm{COHb}$ levels over air quality standard (1.5\% $\mathrm{COHb}$ ) were found in 49 percent of 101 units of banked blood, with the highest $\mathrm{COHb}$ fraction of 12 percent. ${ }^{6}$ The authors concluded that it is not advisable to transfuse such $\mathrm{COHb}$-rich blood to susceptible patients. In another study, fractions up to 9.3 percent $\mathrm{COHb}$ in blood were found, and these authors concluded that number of cigarettes normally smoked every day related to the $\mathrm{COHb}$ fraction. ${ }^{7}$ The suggestion in that study was that all banked blood should be screened and labeled with the measured $\mathrm{COHb}$ fraction. Other studies have found fractions as high as 13.5 percent $\mathrm{COHb}$ in blood from blood donors $^{8}$ and 9.6 percent $\mathrm{COHb}$ in banked blood. ${ }^{9}$ All of these studies give rise to important questions. What are the CO concentrations in banked blood today? Is cigarette smoking still the most important external source for CO? Therefore, we designed this study with the aim to investigate CO concentrations in blood from blood donors in our blood center. A further aim of this study was to clarify the impact of cigarette smoking on CO concentrations in banked blood and, if possible, determine the kinetics of CO in smokers.

\section{MATERIALS AND METHODS}

This study was approved by the Regional Ethic Committee in Umeå, Sweden.

\section{Blood collection}

During September 2006, all blood donors arriving at the blood center at Umeå University Hospital were asked if they would participate in the study. After providing written informed consent, they completed a questionnaire about age, smoking history, living environment, workplace, and wood combustion. The questions in the questionnaire were designed to include possible sources for CO contamination, such as exposure to traffic or incomplete combustion. Elapsed time between cigarette smoking and blood donation was also assessed in the questionnaire. A separate blood sample was taken during the original blood donation, for analysis of $\mathrm{CO}$ and $\mathrm{Hb}$. The samples were collected in 1.8-mL tubes (Vacutainer, Becton Dickinson, Franklin Lakes, NJ) containing $0.13 \mathrm{~mol}$ per L sodium citrate. To elucidate the adequate size of the study group, a pilot study was conducted which showed elevated CO con- centrations in 5 of 40 investigated bags. Based on a minimum of 50 donors with elevated CO concentrations, a study group size of 410 was considered large enough to investigate not only how many donors had increased concentrations of $\mathrm{CO}$ but also which external sources might have contributed to this increase. No donors were excluded from the study. Some donors did not answer the whole questionnaire, but their blood samples were still included in the $\mathrm{CO}$ analysis. All individuals answering specific questions have been included in that specific calculation; the different numbers of responses can be seen in Table 1 .

\section{Analysis}

Analyses of CO concentrations in blood were performed using a method described in a recent report. ${ }^{10}$ In short, samples containing $400 \mu \mathrm{L}$ of blood and a glass bead were put into gas-tight containers. Reagent (saponin and sulfuric acid) was added and the gas phase was injected and analyzed on a gas chromatograph with flame ionization detection fitted with a nickel catalyst. This assay had fine reproducibility (coefficient of variation, 1.5\%) and low detection limit $(12 \mu \mathrm{mol} / \mathrm{L})$. Hb was measured using hemoximetry (OSM3 hemoximeter, Radiometer, Copenhagen, Denmark) and a hematology analyzer (Sysmex K-4500, Sysmex Corp., Chou-ku, Japan). Results from the gas chromatographic method were measured in $\mu$ mol per $\mathrm{L}$ and transformed to $\mathrm{COHb}$ fractions using the $\mathrm{Hb}$ concentration and the formula ${ }^{11}$

$$
\mathrm{COHb}=\frac{\mathrm{C} \cdot 64400}{4 \cdot \mathrm{Hb}}
$$

where $\mathrm{COHb}$ is the carboxyhemoglobin fraction and $\mathrm{C}$ is the CO concentration expressed in mol per L. The constant 64400 is the molecular mass of $\mathrm{Hb}$ in mammals. The 
constant 4 represents the four binding sites of $\mathrm{Hb}$ to $\mathrm{CO}$. $\mathrm{Hb}$ is the $\mathrm{Hb}$ concentration $(\mathrm{g} / \mathrm{L})$.

\section{Blood storage in bags and the $\mathrm{CO}$ concentration}

Blood bags (Baxter International, Inc., Deerfield, IL) at the blood center in Umeå was prepared using $450 \mathrm{~mL}$ of whole blood in $63 \mathrm{~mL}$ of citrate-phosphate-dextrose and $100 \mathrm{~mL}$ of saline-adenine-glucose-mannitol. Blood samples from 10 bags were collected using the aliquots obtained using a tube stripper and analyzed for CO and $\mathrm{Hb}$ on the day of donation. The bags were then stored $\left(+4^{\circ} \mathrm{C}\right)$ in the blood center for 1 week before new blood samples were collected for reanalysis.

\section{CO kinetics after a smoked cigarette}

Blood samples from volunteers were collected before and after they smoked one cigarette (from the volunteer's preferred brand). Blood samples were also drawn 1, 3, and 5 hours after the smoked cigarette. Blood was collected in 1.8-mL tubes (Vacutainer, Becton Dickinson) containing 0.13 mol per L sodium citrate and $\mathrm{CO}$ and $\mathrm{Hb}$ were analyzed. Individual half-life $\left(\mathrm{t}_{1 / 2}\right)$ of $\mathrm{CO}$ was calculated using the formula

$$
\mathrm{t}_{1 / 2}=\frac{\ln 2}{\mathrm{k}_{\mathrm{e} 1}},
$$

where $\mathrm{k}_{\mathrm{el}}$ is the elimination constant calculated from loglinear regression analysis of CO concentration versus time:

$$
\mathrm{k}_{\mathrm{el}}=-\frac{d \ln \mathrm{C}}{d \mathrm{t}} .
$$

\section{Statistical analysis}

The Kolmogorov-Smirnov test was used to test for normality, and a p value of less than 0.05 was used to reject a null hypothesis of normal distribution. Since blood CO concentrations obtained in this study in general do not follow normal distribution (Kolmogorov-Smirnov test, $\mathrm{p}<0.001$ ), nonparametric tests have been used in all of the statistical calculations. Differences between groups were analyzed using the U test (SPSS, Inc., Chicago, IL). For multiple-group comparisons, the Kruskal-Wallis test (SPSS, Inc.) was used. When comparing frequencies in two groups, the chi-square test for independence was used (SPSS, Inc.). When comparing CO concentrations on two occasions, the Wilcoxon signed rank test was used (SPSS, Inc.). A p value of less than 0.05 was considered significant. Receiver operating characteristic (ROC) analysis on the CO concentration was performed using computer software (Analyse-it add-in, Analyse-it Software, Ltd., Leeds, England) for a computer spreadsheet program (Microsoft Excel, Microsoft Corp., Redmond, WA) after grouping the data in smokers versus nonsmokers. ${ }^{12}$ ROC analysis on CO concentrations was also made with regard to recent smoking ( $\leq 3 \mathrm{hr}$ since the last cigarette) versus late smoking ( $>6 \mathrm{hr}$ since the last cigarette). Nonsmokers were also included in the latter group. The group with 3 to 6 hours between smoking and blood donation was discarded because it only consisted of one subject. Pearson's $r$ for the $\mathrm{Hb}$ concentration versus $\mathrm{CO}$ concentration was calculated. Pearson's r was also calculated for the CO concentrations versus $\mathrm{COHb}$ values given by the formula for this transformation (Equation [1]).

\section{RESULTS}

\section{Demographics}

A total of 410 persons participated in the study. The results from the questionnaire are presented in Table 1. The proportion of smokers (cigarette, cigar, and pipe) was similar in the female and the male groups, 8.7 and 9.8 percent, respectively. The number of smokers was higher $(\mathrm{p}<0.01$, Pearson chi-square) with increasing age. The number of smokers in the group under 30 years was 7 percent, whereas 24 percent were smokers in the group over 49 years.

\section{CO concentrations}

Individual blood concentrations of CO are shown in Fig. 1. The majority (94\%) had concentrations below $130 \mu \mathrm{mol}$ per L. The CO concentrations in this group were normally distributed ( $p=0.072$, Kolmogorov-Smirnov test) with a mean of $84.5 \mu \mathrm{mol}$ per $\mathrm{L}$ and a standard error of the mean of 0.8. Six percent of the blood samples were found to have CO concentrations over $130 \mu \mathrm{mol}$ per L, and some samples contained up to $561 \mu \mathrm{mol}$ per L. Transformation

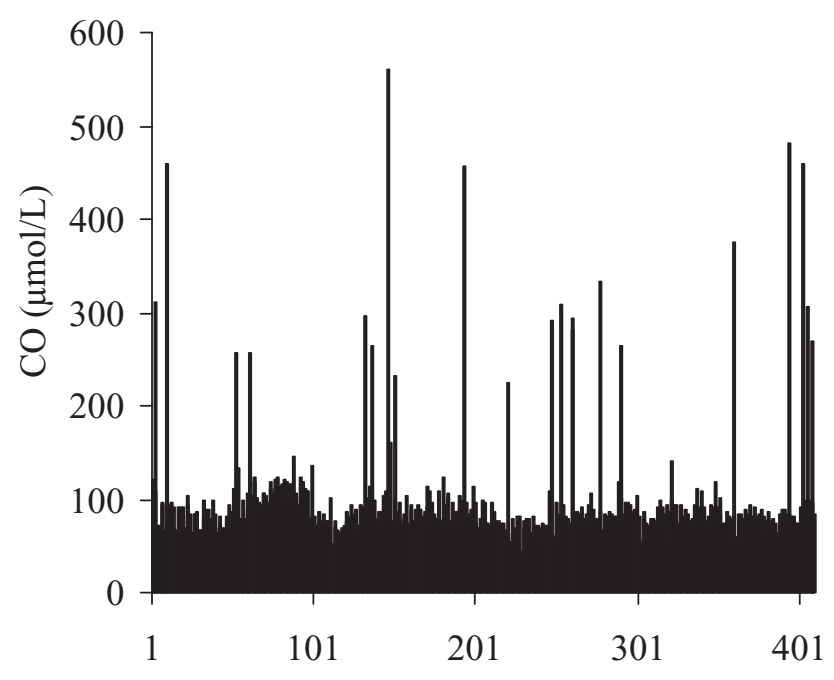

Fig. 1. Individual $(n=410)$ concentrations of $\mathrm{CO}$ in donated blood. 


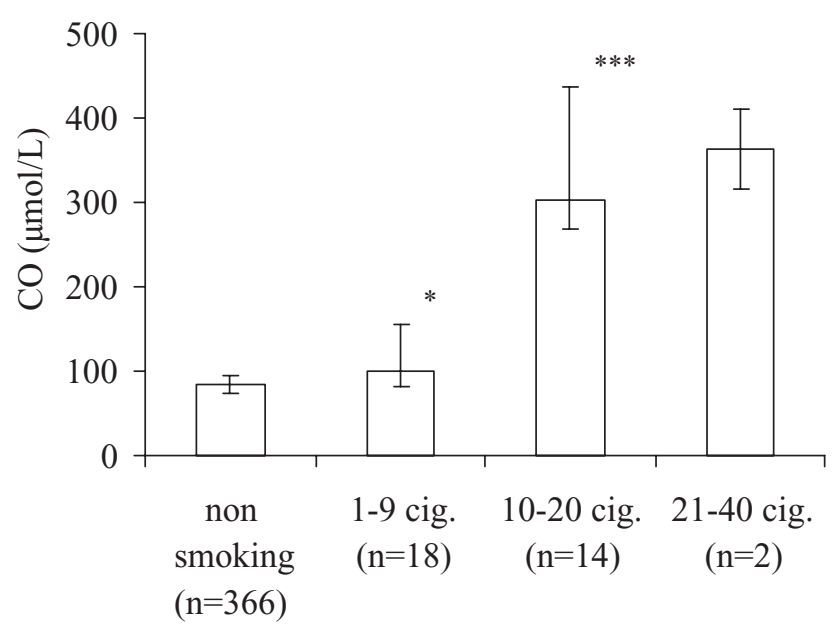

Fig. 2. Median CO concentrations with quartiles grouped by number of consumed cigarettes (cig.) per day for the blood donors. Significant difference at ${ }^{*} \mathbf{p}<0.05,{ }^{* * *} \mathbf{p}<0.001$ versus the nonsmoking group ( $U$ test), as indicated.

of the concentrations according to Equation (1) resulted in a maximum fraction of 5.7 percent $\mathrm{COHb}$. The $\mathrm{CO}$ concentration given in mol per $\mathrm{L}$ and the calculated $\mathrm{COHb}$ values correlated strongly $(\mathrm{r}=0.99)$.

\section{CO in relation to smoking habit}

A significant difference between $\mathrm{CO}$ concentrations in smokers (cigarette, cigar, and pipe) and nonsmokers was found ( $\mathrm{p}<0.001$, $\mathrm{U}$ test). The median concentration was $142 \mu \mathrm{mol}$ per $\mathrm{L}$ (range, $63-561 \mu \mathrm{mol} / \mathrm{L}$ ) or 1.5 percent $\mathrm{COHb}$ (range, $0.8 \%-5.3 \%$ ) in smokers and $83 \mu \mathrm{mol}$ per $\mathrm{L}$ (range, $41-311 \mu \mathrm{mol} / \mathrm{L}$ ) or 0.9 percent $\mathrm{COHb}$ (range, $0.3 \%$ $4.4 \%$ ) in nonsmokers. The group of cigarette smokers had a median CO concentration of $229 \mu \mathrm{mol}$ per L (range, 63-561 $\mu \mathrm{mol} / \mathrm{L}$ ) or 2.4 percent $\mathrm{COHb}$ (range, $0.8 \%-5.3 \%$ ), whereas cigar and pipe smokers had concentrations at levels similar to those of nonsmokers. In Fig. 2, CO concentrations (median and quartiles) grouped by cigarette consumption are shown. A significant difference was detected when nonsmokers were compared with two of the smoking groups; in the third smoking group the number of persons was too small to be included in the statistical calculations.

\section{CO in relation to smoking history}

Figure 3 shows the CO concentration at different time intervals between cigarette smoking and blood donation. There was a significant difference in CO concentration in the group who smoked cigarettes less than 1 hour before the blood donation compared both with the nonsmoking group and with the group with subjects having smoked cigarettes more than 6 hours before leaving the blood sample. In the group who smoked cigarettes 1 to 3 hours

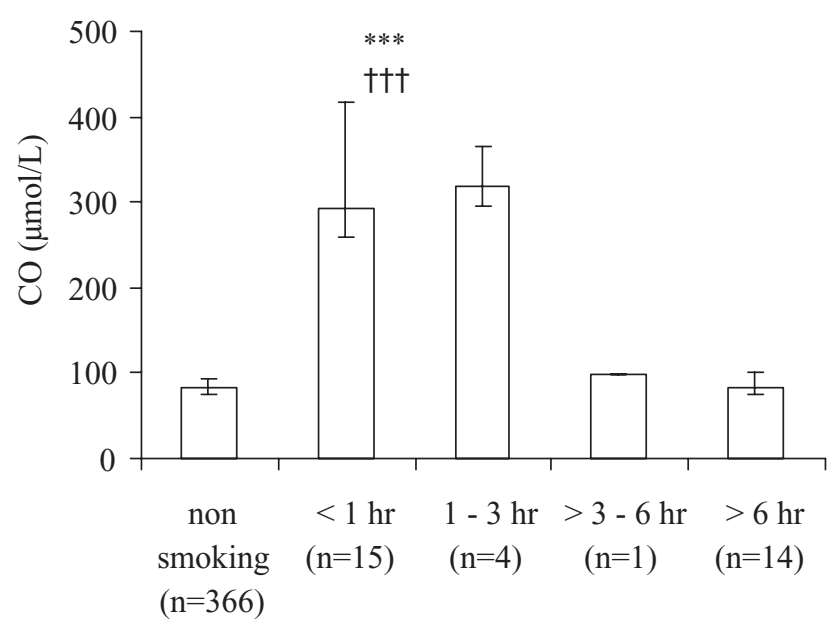

Fig. 3. Median CO concentrations with quartiles, grouped by elapsed time after the last smoked cigarette before blood donation. Significant difference at ${ }^{* * *} \mathbf{p}<0.001$ versus the nonsmoking group and $\dagger+\dagger p<0.001$ versus the group with more than 6 hours since the last cigarette (U test), as indicated.

before blood donation the number of persons is too small to be included in the statistical calculations but the median concentration is similar to that in the group who smoked less than 1 hour before blood donation.

\section{$\mathrm{CO}$ in relation to other factors than smoking}

Comparison of $\mathrm{CO}$ concentration in the nonsmoking group with age and living environment (U test) as well as combustion with wood/pellets and workplace (KruskalWallis) resulted in no differences in concentrations in relation to any of these possible factors. However, male nonsmokers had significantly higher $(\mathrm{p}<0.001$, U test) concentrations of $\mathrm{CO}$ (median, $87 \mu \mathrm{mol} / \mathrm{L}$ ) compared with female nonsmokers (median, $78 \mu \mathrm{mol} / \mathrm{L}$ ). Two nonsmokers of male sex had CO concentrations in the same range as those found in the smoking group (291 and $311 \mu \mathrm{mol} / \mathrm{L})$. After removing these possible outliers, the significant difference $(p<0.001)$ between the sexes remained. Male nonsmokers also had higher $(\mathrm{p}<0.001, \mathrm{U}$ test) $\mathrm{Hb}$ concentrations (median, $147 \mathrm{~g} / \mathrm{L}$ ) compared with female nonsmokers (median, $131 \mathrm{~g} / \mathrm{L}$ ). However, there was no significant difference in $\mathrm{COHb}$ fraction between men and women ( $U$ test). When sex differences in the whole population (smokers and nonsmokers) were compared, the results remained, with a difference in $\mathrm{CO}$ and $\mathrm{Hb}$ concentration between the sexes but no difference in $\mathrm{COHb}$ fraction. There was no significant correlation $(r=0.190)$ between $\mathrm{Hb}$ and $\mathrm{CO}$ concentrations.

\section{ROC analysis}

In the first ROC analysis, a cutoff value of $100 \mu \mathrm{mol}$ per $\mathrm{L}$ CO resulted in a sensitivity of 67.6 percent and a specificity 


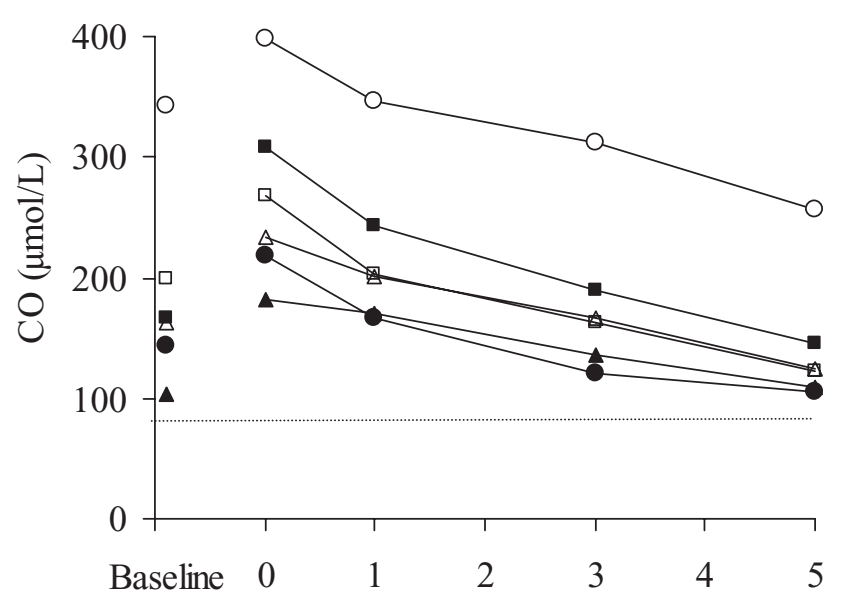

Time after a smoked cigarette (hr)

Fig. 4. Individual $(n=6) \mathrm{CO}$ concentrations in smokers, before and after smoking one cigarette. Baseline $\mathrm{CO}$ concentrations are depicted 10 minutes before cigarette smoking. $(O, \square, \Delta)$ Subjects who had smoked 2 to 3 hours before the baseline sample; $(\Theta, \square, \Delta)$ subjects who had smoked 12 to 13 hours before baseline measurements. $(O)$ An individual donor who smoked 21 to 40 cigarettes every day, whereas the other smokers consumed less. A line representing nonsmokers is also given in the figure and denotes the median CO concentration in 366 nonsmokers analyzed on one occasion.

of 84.4 percent to discriminate between nonsmokers and smokers. At a cutoff value of $135 \mu \mathrm{mol}$ per L CO, the sensitivity was 100 percent and the specificity was 98.9 percent to detect recent cigarette smoking (less than $3 \mathrm{hr}$ ago) in contrast to late cigarette smoking (more than $6 \mathrm{hr}$ ago). Thus, an elevated CO concentration is a very strong indicator of recent cigarette smoking.

\section{Storage of blood bags}

One week of storage, according to the routine procedure, resulted in a median increase of $6 \mu \mathrm{mol}$ per L CO in the blood bags compared with the $\mathrm{CO}$ concentration in the bags measured on the same day as the blood was donated (range of the difference, -13 to $+26 \mu \mathrm{mol} / \mathrm{L}$ ). This difference was not significant ( $p=0.169$, Wilcoxon).

\section{CO elimination kinetics after a smoked cigarette}

Results from six smoking volunteers are shown in Fig. 4. Individual CO concentrations decreased over time after smoking a cigarette. However, $\mathrm{CO}$ concentrations before and 5 hours after smoking a cigarette were never reduced to the same level as the median CO concentration in nonsmoking blood donors. The median elimination half-life was 5.3 hours (range, 4.7-8.4 hr). One individual had consistently higher CO concentrations as well as the longest half-life. This person was a heavier smoker (21-40 cigarettes per day) than the other individuals $(<21$ cigarettes per day).

\section{DISCUSSION}

The present study shows that blood samples from 94 percent of the donors had CO concentrations below $130 \mu \mathrm{mol}$ per L. Mean CO concentration for this group was $84.5 \mu \mathrm{mol}$ per L, which we interpret to be the "normal" level for healthy local subjects. Transformation of $130 \mu \mathrm{mol}$ per L using Equation (1) gives a COHb fraction of 1.5 percent at a $\mathrm{Hb}$ level of $140 \mathrm{~g}$ per L. Another study has described levels below 1.5 percent $\mathrm{COHb}$ in blood bags not to exceed air quality standards. ${ }^{6}$ This also corresponds well to a healthy physiologic level of 0.43 and 1.6 percent $\mathrm{COHb}$ in other populations. ${ }^{13,14}$ In our study, concentrations over $130 \mu \mathrm{mol}$ per L CO could be seen in 6 percent of the studied subjects, some of which demonstrated almost six times the mean level for this group.

It is not known what clinical effect transfused blood with $\mathrm{CO}$ concentrations above the physiologic level may have, if any. In adults, the volume from one blood bank $\mathrm{RBC}$ bag is small in comparison with the total blood volume, so the influence from one bag on the CO concentration in the whole body is probably minimal. Assuming a normal male $(70 \mathrm{~kg})$ with a basal CO concentration of $80 \mu \mathrm{mol}$ per $\mathrm{L}$ and a blood volume of $4.9 \mathrm{~L}$, receiving a transfusion of one blood bag $(250 \mathrm{~mL})$ containing $560 \mu \mathrm{mol}$ per L CO, this would result in a theoretical concentration of $103 \mu \mathrm{mol}$ per $\mathrm{L}$ after transfusion. This is in the range of physiologic concentrations of $\mathrm{CO}$. On the other hand, if a premature child $(<2.8 \mathrm{~kg})$ with a blood volume of less than $250 \mathrm{~mL}$ has a blood exchange with one bag $(250 \mathrm{~mL})$ containing $560 \mu \mathrm{mol}$ per L CO, the theoretical end concentration would be $560 \mu \mathrm{mol}$ per L. In another study investigating if elevated $\mathrm{CO}$ concentrations in blood bags influenced blood $\mathrm{CO}$ in children, the results showed a two- to sevenfold increase in CO concentration in the child after transfusion. ${ }^{15}$ With undeveloped lungs and reduced gas exchange capacity, as can occur in some newborns, there could be a risk of reduced oxygen transport when such blood is used for blood exchange.

The biologic effect of CO is complex. On the one hand, $\mathrm{CO}$ has toxic effects, and on the other hand it can have positive biologic effects; ${ }^{16}$ it is probably a matter of dose. At what concentration $\mathrm{CO}$ is toxic has not yet been determined. In a retrospective study it was stated that a fraction of 23 percent $\mathrm{COHb}$ leads to loss of consciousness. ${ }^{16}$ Effects of $\mathrm{CO}$ in healthy men during exercise can be observed even at low CO fractions $(4.8 \%-21.2 \% \mathrm{COHb}) .{ }^{17}$ Studies on patients with angina pectoris showed that $\mathrm{COHb}$ at fractions from 2.0 to 4.5 percent shortened the time to onset of pain during exercise and also increased the duration of pain. ${ }^{18-21}$ Thus, in various patient groups, 
negative effects of $\mathrm{CO}$ are probably seen at lower $\mathrm{CO}$ concentrations than in healthy persons. The reason for the toxic effects can probably differ between persons based on their conditions. Effects on the central nervous system and in the mitochondria can be the main mechanisms of clinical toxicity in healthy persons, whereas the influence of $\mathrm{CO}$ on oxygen delivery is probably more important in persons with an impaired capacity of oxygen delivery.

In our study we detected concentrations up to $561 \mu \mathrm{mol}$ per L CO in the donor's blood and this finding indicates even higher concentrations in donated units, since the hematocrit (Hct) concentration in the bags is higher than in the donor's blood. A rough calculation to determine the Hct in the blood donor can be done using the $\mathrm{Hb}$ concentration analyzed from the donor's blood $(\mathrm{Hb} \times 0.3=\mathrm{Hct})$. The blood center separates the blood with the goal to achieve 50 to 70 percent Hct in the bags. Assuming that the Hct in the blood unit is 60 percent, this would imply a higher $\mathrm{CO}$ concentration by a factor of 1.1 to 1.7 in the bags compared with the donor's blood. Applying this calculation to our data would imply that 23 percent of the bags theoretically could exceed 1.5 percent $\mathrm{COHb}$, with a highest fraction of 7.2 percent $\mathrm{COHb}$.

Cigarette smoking appears to be the main factor causing increased blood concentrations of CO. The time interval between cigarette smoking and blood donation seems to be a particularly important factor. In our study, we investigated elimination kinetics of $\mathrm{CO}$ after smoking a cigarette in six volunteers and our results give an indication of the elimination time for CO in smokers. Since all six volunteers did not have the same elimination halflife (range, 4.7-8.4 hr), there are probably other factors involved that need to be further investigated. Only six individuals is not an adequate group size and it would be interesting to study the elimination time in smokers in a larger study. In another study, kinetics for smokers have been investigated and the result showed twocompartmental kinetics with half-lives of 1.6 hours from the first compartment and 30.9 hours from the second. ${ }^{13}$ Another study supporting the hypothesis that CO could have a second slower elimination phase showed that former smokers had higher $\mathrm{COHb}$ fractions than nonsmokers. ${ }^{14}$ Yet another study suggests that blood donors should avoid cigarettes 24 hours before blood donation. ${ }^{6}$

The cutoff value from the ROC analysis was chosen to obtain the best sensitivity in this application, since the specificity is of less importance. Choosing a cutoff of $100 \mu \mathrm{mol}$ per L CO for smokers versus nonsmokers resulted in a sensitivity of 67.6 percent, that is, the probability that we correctly will determine smokers as smokers. On the other hand, we have 15.1 percent probability to classify nonsmokers as smokers. The other ROC analysis grouping recent versus late smoking showed that at a cutoff of $135 \mu \mathrm{mol}$ per L, we will classify true recent smokers correctly with 100 percent probability with 1.3 percent risk of determining nonrecent smokers as recent. However, the cutoff from the ROC analysis does not reflect or take into consideration any biologic effects.

CO concentrations in the blood did not correlate with any of the other factors investigated in this study (e.g., workplace, combustion, living environment, and age). In this study, female nonsmokers had lower CO concentrations than male. This is surprising and difficult to explain. Women have higher endogenous production of CO during menstruation. ${ }^{5}$ In contrast, the calculated $\mathrm{COHb}$ fractions do not reveal any sex differences. Since the Hb concentration is higher in men, one could expect this to be the explanation. However, this would implicate that CO correlates with $\mathrm{Hb}$, which is not the case.

During the storage of blood bags, an increase in median CO concentration of $6 \mu \mathrm{mol}$ per $\mathrm{L}$ was found. Since this difference was not significant, the conclusion is that the bags may be stored for 1 week in a refrigerator without changes in $\mathrm{CO}$ concentration. Similar results have been obtained by others. ${ }^{7}$ In a study by Uchida and coworkers, ${ }^{9}$ a decrease in $\mathrm{COHb}$ fraction during storage was observed.

In conclusion, this study shows that a proportion of banked blood (6\%) demonstrated CO concentrations above $130 \mu \mathrm{mol}$ per $\mathrm{L}$, which we consider to be the upper physiologic level, with a highest measured concentration of $561 \mu \mathrm{mol}$ per L. The main source for CO was cigarette smoking. The time interval between smoking and blood donation seems to be a particularly important factor regarding the $\mathrm{CO}$ concentration in blood.

\section{REFERENCES}

1. Piantadosi CA. Biological chemistry of carbon monoxide. Antioxid Redox Signal 2002;4:259-70.

2. Roughton F, Darling R. The effect of carbon monoxide on the oxyhemoglobin dissociation curve. Am J Physiol 1944; 141:17-31.

3. Alonso JR, Cardellach F, Lopez S, Casademont J, Miró O. Carbon monoxide specifically inhibits cytochrome c oxidase of human mitochondrial respiratory chain. Pharmacol Toxicol 2003;93:142-6.

4. Ehlers M, McCloskey D, Devejian NS. Alarming levels of carboxyhemoglobin in a unit of banked blood. Anesth Analg 2003;97:289-90; table of contents.

5. Longo LD. The biological effects of carbon monoxide on the pregnant woman, fetus, and newborn infant. Am J Obstet Gynecol 1977;129:69-103.

6. Aronow WS, O'Donohue WJ Jr, Freygang J, Sketch MH. Carboxyhemoglobin levels in banked blood. Chest 1984;85: 694-5.

7. Aker J. Carboxyhemoglobin levels in banked blood: a comparison of cigarette smokers and non-smokers. AANA J 1987;55:421-6. 
8. Spiess W, Weisshaar D, Vojdanovski B. [Carboxyhaemoglobin concentrations in the recipients of banked blood (author's transl)]. Anasth Intensivther Notfallmed 1980;15: 486-93.

9. Uchida I, Tashiro C, Koo YH, Mashimo T, Yoshiya I. Carboxyhemoglobin and methemoglobin levels in banked blood. J Clin Anesth 1990;2:86-90.

10. Sundin AM, Larsson JE. Rapid and sensitive method for the analysis of carbon monoxide in blood using gas chromatography with flame ionisation detection. J Chromatogr B Analyt Technol Biomed Life Sci 2002;766:115-21.

11. Aberg AM, Hultin M, Abrahamsson P, Larsson JE. Circulatory effects and kinetics following acute administration of carbon monoxide in a porcine model. Life Sci 2004;75: 1029-39.

12. van Erkel AR, Pattynama PM. Receiver operating characteristic (ROC) analysis: basic principles and applications in radiology. Eur J Radiol 1998;27:88-94.

13. Cronenberger C, Mould DR, Roethig HJ, Sarkar M. Population pharmacokinetic analysis of carboxyhaemoglobin concentrations in adult cigarette smokers. Br J Clin Pharmacol 2008;65:30-9.

14. Hart CL, Smith GD, Hole DJ, Hawthorne VM. Carboxyhaemoglobin concentration, smoking habit, and mortality in 25 years in the Renfrew/Paisley prospective cohort study. Heart 2006;92:321-4.
15. Kandall SR, Landaw SA, Thaler MM. Carboxyhemoglobin exchange between donors and recipients of blood transfusions. Pediatrics 1973;52:716-8.

16. Mannaioni PF, Vannacci A, Masini E. Carbon monoxide: the bad and the good side of the coin, from neuronal death to anti-inflammatory activity. Inflamm Res 2006;55: 261-73.

17. Ekblom B, Huot R. Response to submaximal and maximal exercise at different levels of carboxyhemoglobin. Acta Physiol Scand 1972;86:474-82.

18. Anderson EW, Andelman RJ, Strauch JM, Fortuin NJ, Knelson JH. Effect of low-level carbon monoxide exposure on onset and duration of angina pectoris. A study in ten patients with ischemic heart disease. Ann Intern Med 1973; 79:46-50.

19. Aronow WS. Aggravation of angina pectoris by two percent carboxyhemoglobin. Am Heart J 1981;101:154-7.

20. Aronow WS, Isbell MW. Carbon monoxide effect on exercise-induced angina pectoris. Ann Intern Med 1973;79: 392-5.

21. Aronow WS, Stemmer EA, Isbell MW. Effect of carbon monoxide exposure on intermittent claudication. Circulation 1974;49:415-7. 